TRANSACTIONS OF THE

AMERICAN MATHEMATICAL SOCIETY

Volume 358, Number 11, November 2006, Pages 5015-5023

S 0002-9947(06)03895-5

Article electronically published on June 13, 2006

\title{
MULTIPLIER IDEALS OF HYPERPLANE ARRANGEMENTS
}

\author{
MIRCEA MUSTAŢĂ
}

\begin{abstract}
In this note we compute multiplier ideals of hyperplane arrangements. This is done using the interpretation of multiplier ideals in terms of spaces of arcs by Ein, Lazarsfeld, and Mustaţă (2004).
\end{abstract}

\section{INTRODUCTION}

If $X$ is a complex smooth variety, and if $\mathfrak{a} \subseteq \mathcal{O}_{X}$ is a coherent sheaf of ideals on $X$, then for every $\lambda \in \mathbb{Q}_{+}^{*}$ one can define the multiplier ideal of exponent $\lambda$ denoted $\mathcal{I}\left(X, \mathfrak{a}^{\lambda}\right)$. The interest in these ideals comes from the fact that on one hand, they encode properties of the singularities of the subscheme defined by $\mathfrak{a}$, and on the other hand, they appear naturally in vanishing theorems. This led to various applications in recent years, in higher-dimensional birational geometry as well as in local algebra (see Laz for a thorough presentation).

The definition of multiplier ideals is given in terms of a log resolution of singularities for the pair $(X, \mathfrak{a})$. This makes the computation of specific examples quite delicate, so there are not many classes of ideals for which the corresponding multiplier ideals are known. One notable exception is the case of multiplier ideals for monomial ideals in the affine space, which have been computed by Howald using toric methods in How2. Most of the other examples where a general formula is known proceed by reduction to the monomial case under suitable genericity assumptions (see [How1]).

In this note we give a formula for the multiplier ideals of hyperplane arrangements. This is achieved using the description of multiplier ideals in terms of spaces of arcs from ELM].

Let $\mathcal{A}$ be a hyperplane arrangement in a vector space $V \simeq \mathbb{C}^{n}$ with $n \geq 1$, i.e. $\mathcal{A}$ is the (reduced) union of affine hyperplanes $H_{1}, \ldots, H_{d} \subset V$. If $h_{i}$ is an equation defining $H_{i}$, then $\mathcal{A}$ is defined by $f=\prod_{i} h_{i}$, and our goal is to compute $\mathcal{I}\left(V, f^{\lambda}\right)$.

We will assume that $\mathcal{A}$ is central, i.e. that all hyperplanes $H_{i}$ pass through the origin. This is not an important restriction: if $\mathcal{A}$ is an arbitrary arrangement, and if we want to compute multiplier ideals in a neighbourhood of a point $x \in V$, then it is enough to do the computation for the subarrangement consisting of those hyperplanes passing through $x$. Therefore after a suitable translation, we are in the case of a central arrangement.

Received by the editors February 20, 2004 and, in revised form, November 5, 2004

2000 Mathematics Subject Classification. Primary 14B05; Secondary 52C35.

Key words and phrases. Arcs, multiplier ideals, hyperplane arrangements.

The author served as a Clay Mathematics Institute Research Fellow while this research was conducted. 
Let $L(\mathcal{A})$ be the intersection lattice of $\mathcal{A}$ (see OT for basic notions about arrangements). $L(\mathcal{A})$ is the set of all intersections of the hyperplanes in $\mathcal{A}$. If $W \in L(\mathcal{A})$, then its rank is defined by $r(W):=\operatorname{codim}(W)$. We also denote by $s(W)$ the number of hyperplanes in $\mathcal{A}$ containing $W$. Note that $V \in L(\mathcal{A})$, and we put $L^{\prime}(\mathcal{A})=L(\mathcal{A}) \backslash\{V\}$. For every $W \in L(\mathcal{A})$, the ideal defining $W$ in $V$ is denoted by $I_{W}$. If $x$ is a real number, then $\lfloor x\rfloor$ denotes the largest integer $m$ such that $m \leq x$. Our main result is the following.

Theorem 0.1. Let $\mathcal{A}$ be a central hyperplane arrangement defined by $(f=0)$. For every $\lambda \in \mathbb{Q}_{+}^{*}$, we have

$$
\mathcal{I}\left(V, f^{\lambda}\right)=\bigcap_{W \in L^{\prime}(\mathcal{A})} I_{W}^{\lfloor\lambda s(W)\rfloor-r(W)+1} .
$$

Of course, we follow the convention that if $r \leq 0$, then $\left(I_{W}\right)^{r}=\mathcal{O}_{V}$.

Multiplier ideals are characterized by suitable conditions on the orders of vanishing along divisors over $V$. One can interpret the above theorem as saying that in this description it is enough to consider only those divisors which appear as exceptional divisors for the blowings-up of $V$ along the various $W$ in $L^{\prime}(\mathcal{A})$ (see Remark 1.2 below).

Corollary 0.2. With the above notation, the support of the subscheme defined by $\mathcal{I}\left(V, f^{\lambda}\right)$ is equal to the union of those $W \in L^{\prime}(\mathcal{A})$ such that $\lambda \geq \frac{r(W)}{s(W)}$.

An important invariant of an ideal $\mathfrak{a}$ is its $\log$ canonical threshold $\operatorname{lc}(\mathfrak{a})$. It can be defined as the smallest $\lambda$ such that $\mathcal{I}\left(X, \mathfrak{a}^{\lambda}\right) \neq \mathcal{O}_{X}$. We deduce the following formula in the case of a hyperplane arrangement.

Corollary 0.3. If $\mathcal{A}$ is a central hyperplane arrangement defined by $(f=0)$, then

$$
\operatorname{lc}(f)=\min _{W \in L^{\prime}(\mathcal{A})} \frac{r(W)}{s(W)} .
$$

We will prove the above results in the next section, after we review the description of multiplier ideals in terms of spaces of arcs from [ELM]. In the last section we discuss jumping coefficients in the sense of [ELSV] for hyperplane arrangements.

\section{Proof of the main Result}

We denote by $\mathbb{N}$ the set of nonnegative integers. Let us first recall a few basic facts about spaces of arcs. For more details we refer to ELM]. Recall that we work in the ambient smooth variety $V \simeq \mathbb{C}^{n}$. The arc space $V_{\infty}$ of $V$ is the set of all morphisms $\gamma: \operatorname{Spec} \mathbb{C}[[t]] \longrightarrow V$. Since $V=\operatorname{Spec}\left(\operatorname{Sym}\left(V^{*}\right)\right)$, an arc $\gamma$ can be identified with a ring homomorphism $\gamma^{\#}: \operatorname{Sym}\left(V^{*}\right) \longrightarrow \mathbb{C}[[t]]$, hence with a morphism of vector spaces $u: V^{*} \longrightarrow \mathbb{C}[[t]]$. By looking at the coefficients of the different powers of $t$, we may identify $u$ with $\left(u^{(m)}\right)_{m} \in V^{\mathbb{N}}$. Note also that if we fix a basis $e_{1}, \ldots, e_{n}$ for $V$, then giving $u$ is the same as giving an element $\left(u_{i}\right)_{i} \in(\mathbb{C}[[t]])^{n}$, where $u_{i}=\gamma^{\#}\left(e_{i}^{*}\right)$. We will freely use these identifications below.

If $g \in \mathcal{O}(V)=\operatorname{Sym}\left(V^{*}\right)$ and if $\gamma \in V_{\infty}$, then the order $\operatorname{ord}_{\gamma}(g)$ of $g$ along $\gamma$ is defined as the order of $\gamma^{\#}(g) \in \mathbb{C}[[t]]$ (the order of zero is infinity). If $m \in \mathbb{N}$, then the contact locus Cont ${ }^{\geq m}(g)$ is the set of all $\gamma$ such that $\operatorname{ord}_{\gamma}(g) \geq m$.

The space of $m$-jets of $V$ is defined as the set of all morphisms

$$
\delta: \text { Spec } \mathbb{C}[[t]] /\left(t^{m+1}\right) \longrightarrow V .
$$


As above this can be naturally identified with $\left(\mathbb{C}[[t]] /\left(t^{m+1}\right)\right)^{n}$, hence $V_{m} \simeq \mathbb{C}^{(m+1) n}$. Every arc $\gamma \in V_{\infty}$ induces a jet $\gamma_{m} \in V_{m}$ by truncation, and this defines a projection morphism $\psi_{m}: V_{\infty} \longrightarrow V_{m}$. If a subset $C$ of $V_{\infty}$ is of the form $\psi_{m}^{-1}(S)$ for a Zariski closed subset $S$ of $V_{m}$ (such a set is called a closed cylinder), then the codimension $\operatorname{codim}(C)$ of $C$ in $V_{\infty}$ is the codimension of $S$ in $V_{m}$ (it is easy to check that this is independent of $m$ ). In particular, this applies to the above contact loci. If $C$ and $S$ are as above, then $C$ is called irreducible if $S$ is so. Note that every closed cylinder admits a unique decomposition in irreducible components.

We now recall the description of multiplier ideals in terms of contact loci. In fact, it is more convenient to change the indexing of the multiplier ideals. Recall that if $f \in \mathcal{O}(V)$ and if $\lambda \in \mathbb{Q}_{+}^{*}$, then there is $\epsilon>0$ such that $\mathcal{I}\left(V, f^{\lambda}\right)=\mathcal{I}\left(V, f^{t}\right)$ for every $t \in[\lambda, \lambda+\epsilon)$. We also remark that there are only finitely many distinct multiplier ideals of exponent $\leq \lambda$ (for this and other basic properties of multiplier ideals, we refer to [Laz $)$. We put

$$
\widetilde{\mathcal{I}}\left(V, f^{\lambda}\right):=\mathcal{I}\left(V, f^{\lambda-\epsilon}\right),
$$

where $0<\epsilon \ll 1$. It follows that $\mathcal{I}\left(V, f^{\lambda}\right)=\tilde{\mathcal{I}}\left(V, f^{\lambda+t}\right)$ for $0<t \ll 1$. With this notation, Corollary 3.15 in [ELM] can be rewritten as

Theorem 1.1. Let $f \in \mathcal{O}(V)$ be a nonzero element and let $g \in \mathcal{O}(V)$. If $\lambda \in \mathbb{Q}_{+}^{*}$, then $g \in \widetilde{\mathcal{I}}\left(V, f^{\lambda}\right)$ if and only if for every $m$ and every irreducible component $C$ of Cont $^{\geq m}(f)$ we have

$$
\operatorname{ord}_{\gamma}(g) \geq \lambda m-\operatorname{codim}(C)
$$

for all $\gamma \in C$.

We can now give the proof of our main result.

Proof of Theorem 0.1. Using the notation introduced above, proving formula (1) for all $\lambda$ is equivalent to proving that

$$
\tilde{\mathcal{I}}\left(V, f^{\lambda}\right)=\bigcap_{W \in L^{\prime}(\mathcal{A})} I_{W}^{\lceil\lambda s(W)\rceil-r(W)}
$$

for all $\lambda$, where $\lceil x\rceil$ denotes the smallest integer $m$ such that $x \leq m$.

Note first that in order to prove (5), we may assume that $\mathcal{A}$ is an essential arrangement, i.e. that $\{0\} \in L(\mathcal{A})$. Indeed, let $T$ be the intersection of the hyperplanes in $\mathcal{A}$. Then there is an isomorphism $V \simeq T \times V^{\prime}$ and an essential hyperplane arrangement $\mathcal{A}^{\prime}$ in $V^{\prime}$ defined by $f^{\prime}$ such that $\mathcal{A}$ is the pull-back of $\mathcal{A}^{\prime}$ via the canonical projection $\pi: T \times V^{\prime} \longrightarrow V^{\prime}$. As

$$
\widetilde{\mathcal{I}}\left(V, f^{\lambda}\right)=\pi^{-1} \widetilde{\mathcal{I}}\left(V^{\prime},\left(f^{\prime}\right)^{\lambda}\right)
$$

for all $\lambda$, it is clear that it is enough to prove (5) for $\mathcal{A}^{\prime}$. We may therefore assume that $\mathcal{A}$ is essential.

We now use Theorem 1.1 to describe $\widetilde{\mathcal{I}}\left(V, f^{\lambda}\right)$. Recall that we denote by $h_{i}$ an equation of the hyperplane $H_{i}$, so $f=\prod_{i} h_{i}$. It is clear that we have a decomposition

$$
\text { Cont }^{\geq m}(f)=\bigcup_{\sum_{i} a_{i}=m} \bigcap_{i=1}^{d} \operatorname{Cont}^{\geq a_{i}}\left(h_{i}\right)
$$


Consider $u=\left(u^{(j)}\right)_{j \in \mathbb{N}} \in V_{\infty}$, where $u^{(j)} \in V$ for all $j$. Note that $u \in$ Cont $^{\geq a_{i}}\left(h_{i}\right)$ if and only if $u^{(j)} \in H_{i}$ for all $j$ such that $0 \leq j \leq a_{i}-1$. Hence $u \in \bigcap_{i}$ Cont $^{\geq a_{i}}\left(h_{i}\right)$ if and only if for all $j$ we have

$$
u^{(j)} \in \bigcap_{a_{i}>j} H_{i} .
$$

For every $a=\left(a_{1}, \ldots, a_{d}\right) \in \mathbb{N}^{d}$ and every $j \in \mathbb{N}$, consider

$$
W_{j}(a)=\bigcap_{a_{i}>j} H_{i} \in L(\mathcal{A}) .
$$

In this way we get a sequence $W_{\bullet}(a)$ such that $W_{j}(a) \subseteq W_{j+1}(a)$ for every $j$, and $W_{m}(a)=V$ if $m \gg 0$.

It follows from the above description that $\bigcap_{i}$ Cont $^{\geq a_{i}}\left(h_{i}\right)$ is a closed irreducible cylinder of codimension $\sum_{j \in \mathbb{N}} \operatorname{codim} W_{j}(a)$. Theorem 1.1 and the decomposition (66) imply that given a polynomial $g \in \mathcal{O}(V)$, we have $g \in \widetilde{\mathcal{I}}\left(V, f^{\lambda}\right)$ if and only if for every $a_{1}, \ldots, a_{d} \in \mathbb{N}$ we have

$$
\operatorname{ord}_{u}(g) \geq \lambda \cdot \sum_{i=1}^{d} a_{i}-\sum_{j \in \mathbb{N}} \operatorname{codim} W_{j}(a)
$$

for all $u=\left(u^{(j)}\right)_{j \in \mathbb{N}} \in V_{\infty}$ with $u^{(j)} \in W_{j}(a)$ for every $j$.

We now reformulate the above condition starting this time with a sequence $W_{\bullet}$. Suppose that $W_{\bullet}=\left(W_{m}\right)_{m \in \mathbb{N}}$ is a sequence of elements in $L(\mathcal{A})$ such that $W_{j} \subseteq W_{j+1}$ for every $j$, and such that $W_{m}=V$ for $m \gg 0$.

Given $W_{\bullet}$ as above, let $Z\left(W_{\bullet}\right)$ be the set of $\operatorname{arcs} u=\left(u^{(m)}\right)_{m \in \mathbb{N}} \in V_{\infty}$ such that $u^{(j)} \in W_{j}$ for every $j$. We see that $Z\left(W_{\bullet}\right)$ is a closed irreducible cylinder, and if $u$ is a general element in $Z\left(W_{\bullet}\right)$, then

$$
\operatorname{ord}_{u}\left(h_{i}\right)=b_{i}\left(W_{\bullet}\right):=\min \left\{m \in \mathbb{N} \mid W_{m} \not \subset H_{i}\right\} .
$$

Note that if $W_{\bullet}$ corresponds to $a \in \mathbb{N}^{d}$, as before, i.e. if $W_{m}=W_{m}(a)$ for all $m$, then $W_{a_{i}-1} \subseteq H_{i}$ by definition, so $b_{i}\left(W_{\bullet}\right) \geq a_{i}$ for all $i$.

We claim that if we start with $W_{\bullet}$, and if $b=\left(b_{1}\left(W_{\bullet}\right), \ldots, b_{d}\left(W_{\bullet}\right)\right)$, where $b_{i}\left(W_{\bullet}\right)$ is defined by (8), then $W_{j}=W_{j}(b)$ for all $j$. Indeed, let us denote $W_{j}^{\prime}=\bigcap_{b_{i}>j} H_{i}$. By definition, $j<b_{i}$ implies $W_{j} \subseteq H_{i}$, so $W_{j} \subseteq W_{j}^{\prime}$. For the reverse inclusion, since $W_{j} \in L(\mathcal{A})$, it is enough to show that if $W_{j} \subseteq H_{k}$ for some $k$, then $W_{j}^{\prime} \subseteq H_{k}$. But $W_{j} \subseteq H_{k}$ implies $b_{k}>j$, so $W_{j}^{\prime} \subseteq H_{k}$ by definition. Therefore $W_{j}=W_{j}^{\prime}$ for all $j$, which proves our claim.

We now reinterpret the condition in (7). We claim that $g \in \tilde{\mathcal{I}}\left(V, f^{\lambda}\right)$ if and only if for every sequence $W_{\bullet}$ as above we have

$$
\operatorname{ord}_{u}(g) \geq \lambda \cdot \sum_{i=1}^{d} b_{i}-\sum_{j \in \mathbb{N}} \operatorname{codim} W_{j}
$$

for every $u \in Z\left(W_{\bullet}\right)$.

To see this, note first that by the above discussion, if we start with $W \bullet$ and define $b$ by (8), then $W_{j}=W_{j}(b)$ for all $j$, so (9) follows from (7) for $b \in \mathbb{N}^{d}$. Conversely, if we start with $a \in \mathbb{N}^{d}$, if $W_{j}=W_{j}(a)$ for every $j$ and if $b$ is given by (8), then (7) follows from (9) since $b_{i} \geq a_{i}$ for all $i$. 
We now fix a sequence $W_{\bullet}$ as above, and we rewrite condition (9) for this sequence. Let $r_{j}$ denote the codimension of $W_{j}$. We may choose a basis $e_{1}, \ldots, e_{n}$ for $V$ such that for every $j$ we have $W_{j}=\left\langle e_{r_{j}+1}, \ldots, e_{n}\right\rangle$.

In this system of coordinates, we identify an arc $\gamma \in V_{\infty}$ with an element $u=$ $\left(u_{1}, \ldots, u_{n}\right) \in(\mathbb{C}[[t]])^{n}$, where $u_{i}=\gamma^{\#}\left(X_{i}\right)$. Here $X_{i}=e_{i}^{*} \in \mathcal{O}(V)=\operatorname{Sym}\left(V^{*}\right)=$ $\mathbb{C}\left[X_{1}, \ldots, X_{n}\right]$. Therefore $Z\left(W_{\bullet}\right)$ can be described as the set of those $\operatorname{arcs} u=\left(u_{i}\right)_{i}$ such that for all $i$ and $j$ with $r_{j} \geq i$, we have $\operatorname{ord}_{u}\left(X_{i}\right)>j$. Hence $u \in V_{\infty}$ is in $Z\left(W_{\bullet}\right)$ if and only if $\operatorname{ord}_{u}\left(X_{i}\right) \geq \min \left\{j \mid r_{j}<i\right\}$. It follows that in this system of coordinates, the set of those $g \in \mathcal{O}(V)$ which satisfy (9) for $W_{\bullet}$ is the monomial ideal generated by those $X_{1}^{\alpha_{1}} \ldots X_{n}^{\alpha_{n}}$ such that

$$
\sum_{k=1}^{n} \alpha_{k} \cdot \min \left\{j \mid r_{j}<k\right\} \geq \lambda \cdot \sum_{i=1}^{d} b_{i}-\sum_{j \in \mathbb{N}} r_{j} .
$$

Let $p_{i}:=\min \left\{j \mid r_{j}<i\right\}$ for every $i$ with $1 \leq i \leq n$, so we have $p_{1} \geq p_{2} \geq \ldots \geq p_{n}$. We see that $W_{\bullet}$ consists of $p_{n}$ zero subspaces, and of $p_{n-i}-p_{n-i+1}$ subspaces of dimension $i$, for every $1 \leq i \leq n-1$. Hence $\sum_{j \in \mathbb{N}} r_{j}=\sum_{k=1}^{n} p_{k}$, and (10) becomes

$$
\sum_{k=1}^{n}\left(\alpha_{k}+1\right) p_{k} \geq \lambda \cdot \sum_{i=1}^{d} b_{i} .
$$

We put $q_{n}=p_{n}$ and $q_{i}=p_{i}-p_{i+1}$ for $1 \leq i \leq n-1$. We call the sequence $W_{\bullet}$ strict if $\operatorname{dim}\left(W_{j}\right)=j$ for $0 \leq j \leq n$. It is clear that every $W_{\bullet}$ as above is obtained from a strict $W_{\bullet}^{\prime}$ by taking $q_{n-i}$ copies of each $W_{i}^{\prime}$ for $0 \leq i \leq n-1$ (the remaining $W_{i}$ being equal to the ambient space). Note that some of the $q_{i}$ may be zero. In addition, if $b^{\prime}=\left(b_{1}^{\prime}, \ldots, b_{d}^{\prime}\right)$ corresponds to $W_{\bullet}^{\prime}$, then we have $b_{i}=p_{n+1-b_{i}^{\prime}}$ for every $i$. If for every $k$ with $1 \leq k \leq n$ we denote by $\tau_{k}$ the number of elements in $\left\{i \mid b_{i}^{\prime}=k\right\}$, we get

$$
\sum_{i=1}^{d} b_{i}=\sum_{k=1}^{n} \tau_{n+1-k} p_{k}
$$

We now let $W_{\bullet}$ vary for a fixed $W_{\bullet}^{\prime}$, i.e. we allow the $q_{i}$ to vary. It follows from (11) that the set of those $g \in \mathcal{O}(V)$ which satisfy (9) for all such $W$ • is the monomial ideal generated by those $X_{1}^{\alpha_{1}} \ldots X_{n}^{\alpha_{n}}$ such that

$$
\sum_{k=1}^{n}\left(\alpha_{k}+1\right) \cdot \sum_{j=k}^{n} q_{j} \geq \lambda \cdot \sum_{k=1}^{n} \tau_{n+1-k} \cdot \sum_{j=k}^{n} q_{j}
$$

for every $q_{1}, \ldots, q_{n} \in \mathbb{N}$. Equivalently, we have

$$
\sum_{\ell=1}^{k}\left(\alpha_{\ell}+1\right) \geq \lambda \cdot \sum_{\ell=1}^{k} \tau_{n+1-\ell}
$$

for every $k, 1 \leq k \leq n$.

We can reformulate this condition without any reference to the system of coordinates as follows. If $g \in \mathcal{O}(V)$, then $g$ satisfies (9) for all $W$. corresponding to a fixed strict sequence $W_{\bullet}^{\prime}$ if and only if for every $k$ with $1 \leq k \leq n$ we have

$$
g \in I_{W_{n-k}^{\prime}}^{\left\lceil\lambda s_{k}\right\rceil-k}
$$


for every $k$, where $s_{k}=\sum_{\ell=1}^{k} \tau_{n+1-\ell}=s\left(W_{n-k}^{\prime}\right)$. Since every $W \in L(\mathcal{A}), W \neq V$ appears as $W_{n-k}^{\prime}$ for a suitable strict sequence $W_{\bullet}^{\prime}$ (where $k=r(W)$ ), we deduce that $g \in \tilde{\mathcal{I}}\left(V, f^{\lambda}\right)$ if and only if for every $W \in L^{\prime}(\mathcal{A})$ we have

$$
g \in I_{W}^{\lceil\lambda s(W)\rceil-r(W)} .
$$

This completes the proof of the theorem.

Remark 1.2. The multiplier ideals of $f$ are characterized by the orders of vanishing along divisors over $V$. More precisely, suppose that $E$ is a divisor on a smooth variety $V^{\prime}$ such that there is a proper, birational morphism $\pi: V^{\prime} \rightarrow V$. Associated to such $E$ there are two numbers: the order $a_{E}(f)$ of $E$ in the pull-back of $\operatorname{div}(f)$ to $V^{\prime}$, and the order $k_{E}$ of $E$ in the relative canonical divisor $K_{V^{\prime} / V}$. The multiplier ideal $\mathcal{I}\left(f^{\lambda}\right)$ is the set of all $g \in \mathcal{O}(V)$ such that

$$
a_{E}(g)>\lambda a_{E}(f)-k_{E}-1
$$

for all $E$ as above.

We may reinterpret the statement of Theorem 0.1 as saying that if $f$ defines a hyperplane arrangement $\mathcal{A}$, then in the above description of $\mathcal{I}\left(f^{\lambda}\right)$ it is enough to consider only those $E$ which are the exceptional divisors of the blowing-ups of $V$ along the various $W$ in $L^{\prime}(\mathcal{A})$. Indeed, for such $E$ corresponding to $W$, note that $a_{E}(f)=s(W)$ and $k_{E}=r(W)-1$. Moreover, we have $g \in I_{W}^{m}$ if and only if $a_{E}(g) \geq m$.

Proof of Corollaries 0.2 and 0.3 . The description of the support of the subscheme defined by $\mathcal{I}\left(V, f^{\lambda}\right)$ follows immediately from the formula in Theorem 0.1 . We deduce that $\mathcal{I}\left(V, f^{\lambda}\right)=\mathcal{O}_{X}$ if and only if $\lambda<r(W) / s(W)$ for every $W \in L^{\prime}(\mathcal{A})$. This gives the formula for $\operatorname{lc}(f)$.

\section{JUMPING COEFFICIENTS OF HYPERPLANE ARRANGEMENTS}

We have seen that Theorem 0.1 gives a formula for the log canonical threshold of a hyperplane arrangement. In this section we discuss the higher jumping coefficients of multiplier ideals.

Let us recall the definition from ELSV]. If $X$ is a smooth variety and if $\mathfrak{a} \subset \mathcal{O}_{X}$ is a nonzero coherent sheaf of ideals, then the jumping coefficients of $\mathfrak{a}$ are the elements of

$$
\left\{\lambda \in \mathbb{Q}_{+}^{*} \mid \mathcal{I}\left(X, \mathfrak{a}^{\lambda}\right) \neq \mathcal{I}\left(X, \mathfrak{a}^{\lambda-\epsilon}\right) \text { if } 0<\epsilon \ll 1\right\} .
$$

Hence $\operatorname{lc}(\mathfrak{a})$ is the smallest jumping coefficient.

Suppose now that $X=V \simeq \mathbb{C}^{n}$ and that $\mathfrak{a}$ is a principal ideal generated by the nonzero polynomial $f$. We recall two basic facts about the jumping coefficients of $f$ (see [ELSV] for proofs). First, 1 is always a jumping coefficient. Second, if $\lambda>1$, then $\lambda$ is a jumping coefficient if and only if $\lambda-1$ is also. Therefore in order to understand these invariants it is enough to study those contained in $(0,1)$.

The jumping coefficients are also related to the Bernstein polynomial $b_{f} \in \mathbb{C}[s]$. More precisely, if $\lambda$ is a jumping coefficient in $(0,1]$, then $b_{f}(-\lambda)=0$.

The following is an immediate consequence of Theorem 0.1

Corollary 2.1. If $\mathcal{A}$ is a central hyperplane arrangement defined by $(f=0)$, then $\lambda \in \mathbb{Q}_{+}^{*}$ is a jumping coefficient of $f$ if and only if there are $W \in L^{\prime}(\mathcal{A})$ and $m \in \mathbb{N}$ 
such that $\lambda=\frac{r(W)+m}{s(W)}$ and such that

$$
\bigcap_{W^{\prime} \in L^{\prime}(\mathcal{A})} I_{W^{\prime}}^{\left\lceil\lambda s\left(W^{\prime}\right)\right\rceil-r\left(W^{\prime}\right)} \nsubseteq I_{W}^{m+1} .
$$

In fact, we may take the above intersection over only those $W^{\prime} \in L^{\prime}(\mathcal{A})$ containing $W$.

Note that by the above corollary, each $W \in L^{\prime}(\mathcal{A})$ gives some candidates for jumping coefficients (we recall that we consider only those numbers in $(0,1)$ ). However, unlike in the case of the log canonical threshold, it is not at all clear how to interpret the condition that $\lambda$ is a jumping number just in terms of the intersection lattice $L(\mathcal{A})$.

Question 2.2. Given $\lambda \in \mathbb{Q}_{+}^{*}$, does the condition that $\lambda$ is a jumping number for $f$ depend only on the intersection lattice $L(\mathcal{A})$ ?

We now consider several particular cases.

Example 2.3. We say that $\lambda$ is a set-theoretic jumping coefficient for $f$ if the support of the subscheme defined by $\mathcal{I}\left(V, f^{\lambda}\right)$ strictly contains the support of the subscheme defined by $\mathcal{I}\left(V, f^{\lambda-\epsilon}\right)$ for $0<\epsilon \ll 1$. The smallest such invariant is the $\log$ canonical threshold.

As in the case of the log canonical threshold, these numbers are easy to describe: $\lambda$ is a set-theoretic jumping coefficient of $f$ if and only if there is $W \in L^{\prime}(\mathcal{A})$ such that $\lambda=\frac{r(W)}{s(W)}$ and such that $\frac{r(W)}{s(W)} \leq \frac{r\left(W^{\prime}\right)}{s\left(W^{\prime}\right)}$ for every $W^{\prime} \in L^{\prime}(\mathcal{A})$ containing $W$. This follows immediately from the description in Corollary 0.2 of the support of the subscheme defined by $\mathcal{I}\left(V, f^{\lambda}\right)$.

Example 2.4. Suppose that $\mathcal{A}$ is a generic arrangement of $d \geq n$ hyperplanes in $\mathbb{C}^{n}$. This means that for every $k \leq n$, the intersection of any $k$ of the hyperplanes in $\mathcal{A}$ has dimension $n-k$. Let $f$ be an equation for $\mathcal{A}$. Walther proved in Wal that the roots of the Bernstein polynomial $b_{f}(s)$ of $f$ are given by $-\frac{n+i}{d}$, with $0 \leq i \leq 2 d-2-n$. We see that the negatives of the roots in $[-1,0)$ are precisely the jumping numbers in $(0,1]$. Indeed, if $\mathbf{m}=\left(X_{1}, \ldots, X_{n}\right)$ and if $\lambda \leq 1$, then it follows from Theorem 0.1 that

$$
\mathcal{I}\left(\mathbb{C}^{n}, f^{\lambda}\right)=\mathbf{m}^{\lfloor\lambda d\rfloor-n+1}
$$

Example 2.5. Let $\mathcal{A}$ be a central arrangement, and consider $W \in L(\mathcal{A})$ with $r(W)=2$. For every $W^{\prime} \in L^{\prime}(\mathcal{A})$ strictly containing $W, W^{\prime}$ has to be one of the hyperplanes, so $r\left(W^{\prime}\right)=s\left(W^{\prime}\right)=1$. It follows from Corollary 2.1 that all candidates for jumping coefficients $\leq 1$ corresponding to $W$

$$
\frac{2+m}{s(W)} \text { for } 0 \leq m \leq s(W)-2
$$

are indeed jumping coefficients. Of course, this is interesting only if $s(W) \geq 3$.

Example 2.6. Suppose now that $W \in L(\mathcal{A})$ has codimension three, and let us analyse when some of the candidates for jumping coefficients corresponding to $W$ are indeed jumping coefficients. It follows from Corollary 2.1 that in order to decide this it is enough to consider in $V / W$ the arrangement induced by those hyperplanes in $\mathcal{A}$ which contain $W$. We may therefore assume that $\operatorname{dim} V=3$ and that $W=(0)$. Since $\mathcal{A}$ is a central arrangement, it is convenient to also consider 
the induced arrangement $\overline{\mathcal{A}}$ in $\mathbf{P}(V) \simeq \mathbf{P}^{2}$. If $P \in \overline{\mathcal{A}}$ is a point in $L(\overline{\mathcal{A}})$ we still denote by $s(P)$ the number of lines in $\overline{\mathcal{A}}$ passing through $P$.

Let $\mathcal{T}_{0}(\mathcal{A})$ be the set of jumping coefficients of $\mathcal{A}$ in $(0,1)$ corresponding to codimension two elements in $L(\mathcal{A})$ :

$$
\mathcal{T}_{0}(\mathcal{A}):=\left\{\frac{j}{s(W)} \mid 2 \leq j \leq s(W)-1, r(W)=2\right\} .
$$

Let $\lambda \in(0,1)$ be a candidate corresponding to the zero subspace, hence $\lambda=j / d$, where $d$ is the number of hyperplanes in $\mathcal{A}$ and $3 \leq j \leq d-1$. We may assume that $\lambda \notin \mathcal{T}_{0}(\mathcal{A})$, and we want to determine whether $\lambda$ is a jumping coefficient. It follows from Corollary 2.1 that $\lambda$ is a jumping coefficient if and only if there is a hypersurface in $\mathbf{P}(V)$ of degree $\leq(j-3)$ passing through each point $P$ in $L(\overline{\mathcal{A}})$ with multiplicity $\geq\lceil j s(P) / d\rceil-2$.

Suppose first that $j=3$. We see that $3 / d$ is a jumping coefficient if and only if for every point $P \in L(\overline{\mathcal{A}})$ we have $s(P) \leq 2 d / 3$.

Suppose now that $j=4$. It follows from the above discussion that $4 / d$ is a jumping coefficient if and only if there is no point $P \in L(\overline{\mathcal{A}})$ with $s(P)>3 d / 4$, and if there are no three noncolinear points $Q_{1}, Q_{2}, Q_{3}$ with $s\left(Q_{i}\right)>d / 2$ for all $i$. However, it is easy to see that if $s\left(Q_{1}\right), s\left(Q_{2}\right)>d / 2$, then $\overline{\mathcal{A}}$ consists precisely of $(d+1) / 2$ lines passing through $Q_{1}$ and $(d+1) / 2$ lines passing through $Q_{2}$, and the line joining $Q_{1}$ and $Q_{2}$ belongs to $\overline{\mathcal{A}}$. In particular, $s(P)=2$ for every other point $P \in L(\overline{\mathcal{A}})$. We conclude that $4 / d$ is a jumping coefficient of $\mathcal{A}$ if and only if for every point $P \in L(\mathcal{A})$ we have $s(P) \leq 3 d / 4$.

Now consider the case $j=5$. We see that $5 / d$ is a jumping coefficient if and only if there is a conic passing through each point $P \in L(\overline{\mathcal{A}})$ with multiplicity $\geq\lceil 5 s(P) / d\rceil-2$. In order for this to be true, we clearly need that $s(P) \leq 4 d / 5$ for every point $P \in L(\overline{\mathcal{A}})$. We show that this is also sufficient. Note first that we cannot have two points $P_{1}, P_{2} \in L(\overline{\mathcal{A}})$ with $s\left(P_{1}\right), s\left(P_{2}\right)>3 d / 5$ or four points $Q_{1}, \ldots, Q_{4}$ with $s\left(Q_{i}\right)>2 d / 5$ for all $i$. Therefore the only case we need to consider is when we have two points $P_{1}, P_{2} \in L(\overline{\mathcal{A}})$ with $s\left(P_{1}\right)>3 d / 5$ and $s\left(P_{2}\right)>2 d / 5$. Again, it is easy to see that in this case every line passes through either $P_{1}$ or $P_{2}$, and the line $\ell$ joining $P_{1}$ and $P_{2}$ is in $\overline{\mathcal{A}}$. In particular, $s(Q)=2$ for every other point $Q$. In this case we may take the conic to be the union of $\ell$ and another line in $\mathcal{A}$ passing through $P_{1}$. We conclude that $5 / d$ is a jumping coefficient if and only if $s(P) \leq 4 d / 5$ for every point $P \in L(\overline{\mathcal{A}})$.

We now consider the case $j=d-1$. We see that $(d-1) / d$ is a jumping coefficient if and only if there is a hypersurface of degree $\leq(d-4)$ passing through each point $P \in L(\overline{\mathcal{A}})$ with multiplicity $\geq s(P)-2$ (we use the fact that $s(P)<d$ for every such $P)$. It is clear that this is not possible if there is $P \in L(\overline{\mathcal{A}})$ such that $s(P)=d-1$. Conversely, if this is not the case, then it is easy to see that we can find four lines in $\overline{\mathcal{A}}$, no three of them meeting in one point. We may take the hypersurface as the union of the remaining $d-4$ lines. Hence $(d-1) / d$ is a jumping coefficient if and only if there is no $P \in L(\overline{\mathcal{A}})$ with $s(P)=d-1$.

Question 2.7. With the notation in the above example, let $\lambda=\frac{j}{d}$ with $3 \leq j \leq$ $d-1$, and suppose that $\lambda \notin \mathcal{T}_{0}(\mathcal{A})$. Is it true that $\lambda$ is a jumping number if and only if for every point $P \in \overline{\mathcal{A}}$ we have $s(P) \leq \frac{(j-1) d}{j}$ ? The above example shows that this is the case if $j \in\{3,4,5, d-1\}$. Note also that in each of these cases, we can choose the relevant hypersurface as a union of lines in $\overline{\mathcal{A}}$. 


\section{ACKNOWLEDGEMENTS}

We are grateful to Zach Teitler for his comments on a previous version of this paper, and to the referee for pointing out Remark [1.2,

\section{REFERENCES}

[ELM] L. Ein, R. Lazarsfeld and M. Mustaţă, Contact loci in arc spaces, Compos. Math. 140 (2004), 1229-1244. MR2081163 (2005f:14006)

[ELSV] L. Ein, R. Lazarsfeld, K. E. Smith and D. Varolin, Jumping coefficients of multiplier ideals, Duke Math. J. 123 (2004), 469-506. MR2068967

[How1] J. Howald, Multiplier ideals of sufficiently general polynomials, preprint, math.AG/0303203.

[How2] J. Howald, Multiplier ideals of monomial ideals, Trans. Amer. Math. Soc. 353 (2001), 2665-2671. MR1828466 (2002b:14061)

[Laz] R. Lazarsfeld, Positivity in algebraic geometry II, Ergebnisse der Mathematik und ihrer Grenzgebiete. 3. Folge, A series of Modern Surveys in Mathematics, Vol. 49, SpringerVerlag, Berlin, 2004.

[OT] P. Orlik and H. Terao, Arrangements of hyperplanes, Grundlehren der Mathematischen Wissenschaften, Vol. 300, Springer-Verlag, New York, 1992. MR1217488 (94e:52014)

[Wal] U. Walther, Bernstein-Sato polynomial versus cohomology of the Milnor fiber for generic hyperplane arrangements, Compos. Math., 41 (2005), 121-145. MR2099772

Department of Mathematics, University of Michigan, Ann Arbor, Michigan 48109

E-mail address: mmustata@umich.edu 